\title{
Mathematics in Natural Science
}

\section{Subordination properties of $p$-valent functions involving the generalized hypergeometric functions}

\author{
Anessa Oshah $^{\mathrm{a}}$, Maslina Darus ${ }^{\mathrm{b}, *}$ \\ ${ }^{a}$ Department of Mathematics, Sabratha University, Libya. \\ ${ }^{b}$ Faculty of Science and Technology, Universiti Kebangsaan Malaysia, 43600 Bangi, Selangor, Malaysia. \\ Communicated by 0. K. Matthew
}

\begin{abstract}
In the present paper, using principle of differential subordination, we investigate some interesting properties of certain subclasses of $p$-valent functions which are defined by linear operator involving the generalized hypergeometric functions.
\end{abstract}

Keywords: Differential subordination, p-valent functions, Hadamard product, hypergeometric functions.

2010 MSC: 30C45, 30C50.

(C)2018 All rights reserved.

\section{Introduction}

Let $\mathcal{A}_{\mathrm{p}}$ denote the class of functions of the form

$$
f(z)=z^{p}+\sum_{k=p+1}^{\infty} a_{k} z^{k}, \quad(p \in \mathbb{N}=\{1,2, \cdots\}),
$$

which are analytic and p-valent in the open unit disc $\mathbb{U}=\{z \in \mathbb{C}:|z|<1\}$, and let $\mathcal{A}_{1}=\mathcal{A}$ a well-known class of normalized analytic functions in $\mathbb{U}$.

Given two functions $f, g \in \mathcal{A}_{p}, f(z)=z^{p}+\sum_{k=p+1}^{\infty} a_{k} z^{k}$ and $g(z)=z^{p}+\sum_{k=p+1}^{\infty} b_{k} z^{k}$ their Hadamard product (convolution) $\mathrm{f}(z) * \mathrm{~g}(z)$ is defined by

$$
f(z) * g(z)=z^{p}+\sum_{k=p+1}^{\infty} a_{k} b_{k} z^{k}, \quad(z \in \mathbb{U}) .
$$

\footnotetext{
*Corresponding author

Email addresses: anessa.oshah@yahoo.com (Anessa Oshah), maslina@ukm.edu.my (Maslina Darus)

doi: $10.22436 / \mathrm{mns} .02 .01 .03$

Received: 2017-06-04 Revised: 2017-10-16 Accepted: 2018-04-11
} 
If $f$ and $g$ are analytic functions in $\mathbb{U}$, we say that $f$ is subordinate to $g$, (or $g$ is superordinate to $f$ ), and write $f(z) \prec g(z)(z \in \mathbb{U})$, if there exists the Schwarz function $w(z)$, analytic in $\mathbb{U}$, with $w(0)=0$ and $|w(z)|<1$ then $f(z)=g(w(z))(z \in \mathbb{U})$. In particular if $g$ is univalent in $\mathbb{U}$ then $f(z) \prec g(z)$ is equivalent to $f(0)=g(0)$ and $f(\mathbb{U}) \subset g(\mathbb{U})$.

For parameters $\alpha_{i} \in \mathbb{C}(i=1, \ldots, q)$, and $\beta_{j} \in \mathbb{C} \backslash\{0,-1,-2, \ldots\}.(j=1, \ldots, s)$, the generalized hypergeometric functions ${ }_{q} F_{s}\left(\alpha_{1}, \ldots, \alpha_{q} ; \beta_{1}, \ldots, \beta_{s} ; z\right)$ is defined as:

$$
\begin{aligned}
&{ }_{\mathrm{q}} F_{s}\left(\alpha_{1}, \ldots, \alpha_{\mathrm{q}} ; \beta_{1}, \ldots, \beta_{s} ; z\right)=\sum_{k=0}^{\infty} \frac{\left(\alpha_{1}\right)_{k}, \ldots,\left(\alpha_{\mathrm{q}}\right)_{\mathrm{k}}}{\left(\beta_{1}\right)_{\mathrm{k}}, \ldots,\left(\beta_{\mathrm{s}}\right)_{\mathrm{k}}} \frac{z^{\mathrm{k}}}{\mathrm{k} !}, \\
&\left(\mathrm{q} \leqslant \mathrm{s}+1, \mathrm{q}, \mathrm{s} \in \mathbb{N}_{0}=\mathbb{N} \cup\{0\}, z \in \mathbb{U}\right),
\end{aligned}
$$

where $(v)_{k}$ is the Pochhammer symbol (or the shifted factorial) defined (in terms of the Gamma function) by

$$
(v)_{k}=\frac{\Gamma(v+k)}{\Gamma(v)}= \begin{cases}1, & k=0, v \in \mathbb{C} \backslash\{0\} \\ v(v+1)(v+2) \ldots . .(v+k-1), & k \in \mathbb{N}=\{1,2,3, \ldots\}\end{cases}
$$

Dziok and Srivastava [4] defined the linear operator under the multivalent analytic functions

$$
\mathrm{H}_{\mathrm{p}}\left(\alpha_{1}, \ldots, \alpha_{\mathrm{q}} ; \beta_{1}, \ldots, \beta_{\mathrm{s}} ; z\right) \mathrm{f}(z): \mathcal{A}_{\mathrm{p}} \rightarrow \mathcal{A}_{\mathrm{p}},
$$

defined by the convolution

$$
\begin{aligned}
H_{p}\left(\alpha_{1}, \ldots, \alpha_{q} ; \beta_{1}, \ldots, \beta_{s} ; z\right) f(z) & =z^{p}{ }_{q} F_{s}\left(\alpha_{1}, \ldots, \alpha_{q} ; \beta_{1}, \ldots, \beta_{s} ; z\right) * f(z) \\
& =z^{p}+\sum_{k=p+1}^{\infty} \Upsilon_{s}^{q, p} a_{k} z^{k}
\end{aligned}
$$

where

$$
\Upsilon_{s}^{q, p}=\frac{\left(\alpha_{1}\right)_{k-p}, \ldots,\left(\alpha_{q}\right)_{k-p}}{\left(\beta_{1}\right)_{k-p}, \ldots,\left(\beta_{s}\right)_{k-p}(k-p) !}
$$

For convenience, we write

$$
H_{p}\left(\alpha_{1}, \ldots, \alpha_{q} ; \beta_{1}, \ldots, \beta_{s} ; z\right) f(z)=H_{q, s}^{p}\left[\alpha_{i}, \beta_{j}\right] f(z) .
$$

Let $\mathcal{M}_{\lambda_{1}, \lambda_{2}, \ell, d}^{\mathrm{m}, \mathrm{d}} \in \mathcal{A}_{\mathrm{p}}$ be defined by

$$
\mathcal{M}_{\lambda_{1}, \lambda_{2}, \ell, d}^{m, p}(z)=z^{p}+\sum_{k=p+1}^{\infty}\left[\frac{\ell\left(p+\left(\lambda_{1}+\lambda_{2}\right)(k-p)\right)+d}{\ell\left(p+\lambda_{2}(k-p)\right)+d}\right]^{m} z^{k}, \quad p \in \mathbb{N},
$$

where $p \in \mathbb{N}, m, d \in \mathbb{N}_{0}=\{0,1,2, \ldots\}, \lambda_{2} \geqslant \lambda_{1} \geqslant 0, \ell \geqslant 0$, and $\ell p+d>0$.

Corresponding to $H_{q, s}^{p}\left[\alpha_{i}, \beta_{j}\right] f(z), \mathcal{M}_{\lambda_{1}, \lambda_{2}, \ell, d}^{m}$ and using Hadamard product, we define a new generalized hypergeometric derivative operator $\mathcal{D}_{\lambda_{1}, \lambda_{2}, \ell, d}^{m, d}\left[\alpha_{i}, \beta_{j}\right]$ as follows:

Definition 1.1. Let the function $f \in \mathcal{A}_{p}$, then the generalized hypergeometric derivative operator

$$
\mathcal{D}_{\lambda_{1}, \lambda_{2}, \ell, \mathrm{d}}^{\mathrm{m}, \mathrm{d}}\left[\alpha_{i}, \beta_{\mathrm{j}}\right]: \mathcal{A}_{\mathrm{p}} \rightarrow \mathcal{A}_{\mathrm{p}}
$$

is given by

$$
\mathcal{D}_{\lambda_{1}, \lambda_{2}, \ell, d}^{m, p}\left[\alpha_{i}, \beta_{j}\right] f(z)=H_{q, s}^{p}\left[\alpha_{i}, \beta_{j}\right] f(z) * \mathcal{M}_{\lambda_{1}, \lambda_{2}, \ell, d}^{m, p}(z)
$$

Then from (1.3) and (1.5) we get

$$
\mathcal{D}_{\lambda_{1}, \lambda_{2}, \ell, d}^{m, p}\left[\alpha_{i}, \beta_{j}\right] f(z)=z^{p}+\sum_{k=p+1}^{\infty}\left[\frac{\ell\left(p+\left(\lambda_{1}+\lambda_{2}\right)(k-p)\right)+d}{\ell\left(p+\lambda_{2}(k-p)\right)+d}\right]^{m} \Upsilon_{s}^{q, p} a_{k} z^{k} .
$$


It follows from the above definition that

$$
\begin{gathered}
{\left[\ell\left(p+\lambda_{2}(k-p)\right)+d\right] \mathcal{D}_{\lambda_{1}, \lambda_{2}, \ell, d}^{m+1, p}\left[\alpha_{i}, \beta_{j}\right] f(z)=\left[\ell\left(p+\lambda_{2}(k-p)-p \lambda_{1}\right)+d\right] \mathcal{D}_{\lambda_{1}, \lambda_{2}, \ell, d}^{m, p}\left[\alpha_{i}, \beta_{j}\right] f(z)} \\
+\ell \lambda_{1} z\left(\mathcal{D}_{\lambda_{1}, \lambda_{2}, \ell, d}^{m, p}\left[\alpha_{i}, \beta_{j}\right] f(z)\right)^{\prime}, \\
\alpha_{i} \mathcal{D}_{\lambda_{1}, \lambda_{2}, \ell, d}^{m, p}\left[\alpha_{i}+1, \beta_{j}\right] f(z)=z\left(\mathcal{D}_{\lambda_{1}, \lambda_{2}, \ell, d}^{m, p}\left[\alpha_{i}, \beta_{j}\right] f(z)\right)^{\prime}+\left(\alpha_{i}-p\right) \mathcal{D}_{\lambda_{1}, \lambda_{2}, \ell, d}^{m, p}\left[\alpha_{i}, \beta_{j}\right] f(z), \quad(i=1,2, \cdots, q) .
\end{gathered}
$$

Remark 1.2. It should be remarked that $\mathcal{D}_{\lambda_{1}, \lambda_{2}, \ell, d}^{0, p}\left[\alpha_{i}, \beta_{j}\right] f(z)=H_{q, s}^{p}\left[\alpha_{i}, \beta_{j}\right] f(z)$ where the linear operator $\mathrm{H}_{\mathrm{q}, \mathrm{s}}^{\mathrm{p}}\left[\alpha_{i}, \beta_{\mathrm{j}}\right] \mathrm{f}(z)$ was introduced by Dziok and Srivastava [4]. Also, the linear operator $\mathcal{D}_{\lambda_{1}, \lambda_{2}, \ell, \mathrm{d}}^{\mathrm{m}, \mathrm{p}}\left[\alpha_{i}, \beta_{\mathrm{j}}\right] \mathrm{f}(z)$ includes various other linear operators which were considered in earlier works. We list a few of them.

(i) For $\ell=1$, we get the operator $\mathcal{D}_{\lambda_{1}, \lambda_{2}, p}^{m, b}$ given by El-Yagubi [7].

(ii) For $q=s+1, \alpha_{i}=1(i=1, \cdots, s+1), \beta_{j}=1(j=1, \cdots, s), \lambda_{2}=d=0, \ell=\lambda_{1}=1$, we get the operator $\mathrm{I}_{p}(\mathrm{~m}, \ell)$ given by Kumar et al. [9].

(iii) For $q=s+1, \alpha_{i}=1(i=1, \cdots, s+1), \beta_{j}=1(j=1, \cdots, s), \lambda_{2}=0$ and $\ell=1$, we get the operator $\mathrm{I}_{\mathrm{p}}^{\mathrm{m}}(\lambda, \ell)$ given by Cătąs [2].

(iv) For $q=s+1, \alpha_{i}=1(i=1, \cdots, s+1), \beta_{j}=1(j=1, \cdots, s), \lambda_{2}=d=0, p=1$ and $\lambda_{1}=\ell=1$, we get salagean operator $\mathrm{D}^{\mathrm{m}}[13]$.

(v) For $q=s+1, \alpha_{i}=1(i=1, \cdots, s+1), \beta_{j}=1(j=1, \cdots, s), \lambda_{2}=d=0, p=1$ and $\ell=1$, we get the operator $\mathrm{D}_{\lambda}^{\mathrm{m}}$ given by Al-Oboudi [1].

(vi) For $\mathrm{q}=2, \mathrm{~s}=1, \alpha_{1}=\mathrm{n}+1, \alpha_{2}=1$ and $\beta_{1}=1, \mathrm{p}=1$, we get derivative operator $\mathcal{D}_{\lambda_{1}, \lambda_{2}, \ell, \mathrm{d}}^{\mathrm{n}, \mathrm{m}}$ given by Oshah and Darus [11].

(vii) For $\mathrm{q}=2, \mathrm{~s}=1, \alpha_{1}=\alpha+1, \alpha_{2}=1, \beta_{1}=1, \ell=1, \mathrm{p}=1$ and $\mathrm{d}=0$, we get derivative operator $\mathrm{D}_{\lambda_{1}, \lambda_{2}}^{\mathrm{m}, \alpha}$ given by Eljamal and Darus [5].

(viii) For $\mathrm{q}=2, \mathrm{~s}=1, \alpha_{1}=\delta+1, \alpha_{2}=1, \beta_{1}=1, \mathrm{p}=1$ and $\ell=1$, we get derivative operator $\mathrm{D}_{\lambda_{1}, \lambda_{2}, \delta}^{\mathrm{m}, \mathrm{b}}$ given by El-Yagubi and Darus [6].

(ix) For $\mathrm{q}=2, \mathrm{~s}=1, \alpha_{1}=\mathrm{n}+1, \alpha_{2}=1, \beta_{1}=1, \lambda_{1}=1$ and $\lambda_{2}=0$, we get derivative operator $\mathrm{I}_{\alpha, \beta}^{\mathrm{m}}$ given by Swamy [15]. In the case when $p=1, \mathrm{I}_{\alpha, \beta}^{\mathrm{m}}$ is the derivative operator defined also by Swamy [14].

(x) For $\mathrm{q}=\mathrm{s}+1, \lambda_{2}=0, \mathrm{p}=1$ and $\lambda_{1}=\ell=1, \mathrm{~d}=\lambda$, we get derivative operator $\mathrm{I}_{\lambda}^{\mathrm{m}}$ given by Cho and Srivastava [3].

Now, for $\mathrm{f}(z) \in \mathcal{A}_{\mathrm{p}}$, the integral operator $\mathrm{I}_{\mu, \mathrm{p}}$ defined by

$$
\left.I_{\mu, p}(z)=I_{\mu, p}(f)(z)\right)=\frac{\mu+p}{z^{\mu}} \int_{0}^{z} t^{\mu-1} f(t) d t, \quad(\mu>-p) .
$$

It can easily be verified from (1.9) that

$$
z\left(\mathcal{D}_{\lambda_{1}, \lambda_{2}, \ell, d}^{m, p}\left[\alpha_{i}, \beta_{j}\right] I_{\mu, p}(z)\right)^{\prime}=(\mu+p) \mathcal{D}_{\lambda_{1}, \lambda_{2}, \ell, d}^{m, p}\left[\alpha_{i}, \beta_{j}\right] f(z)-\mu \mathcal{D}_{\lambda_{1}, \lambda_{2}, \ell, d}^{m, p}\left[\alpha_{i}, \beta_{j}\right] I_{\mu, p}(z) .
$$

\section{A set of preliminary lemmas}

To prove our main result, we need the following lemmas. 
Lemma 2.1 ([8]). Let $\mathrm{h}$ be a convex function with $\mathrm{h}(0)=1$ and let $\gamma \in \mathbb{C} \backslash\{0\}$ be a complex number with $\mathfrak{R e}\{\gamma\} \geqslant 0$. Suppose also that the function $\mathrm{p}$ given by

$$
p(z)=1+p_{1}+p_{2} z^{2}+\cdots,
$$

is analytic in $\mathbb{U}$. If

$$
p(z)+\frac{z p^{\prime}(z)}{\gamma} \prec h(z),(z \in \mathbb{U})
$$

then

$$
\mathrm{p}(z) \prec \mathrm{q}(z) \prec \mathrm{h}(z),(z \in \mathbb{U})
$$

where

$$
\mathrm{q}(z)=\gamma z^{-\gamma} \int_{0}^{z} \mathrm{~h}(\mathrm{t}) \mathrm{t}^{\gamma-1} \mathrm{dt},(z \in \mathbb{U}) .
$$

The function $\mathrm{q}$ is convex and is the best dominant.

For real or complex numbers $\alpha_{1}, \alpha_{2}, \beta_{1}\left(\beta_{1} \neq 0,-1,-2, \cdots\right)$ the hypergeometric function is defined by

$$
{ }_{2} \mathrm{~F}_{1}\left(\alpha_{1}, \alpha_{2} ; \beta_{1} ; z\right)=1+\frac{\alpha_{1} \alpha_{2}}{\beta_{1}} \frac{z}{1 !}+\frac{\alpha_{1}\left(\alpha_{1}+1\right) \alpha_{2}\left(\alpha_{2}+1\right)}{\beta_{1}\left(\beta_{1}+1\right)} \frac{z^{2}}{2 !}+\cdots
$$

We note the series in (2.2) converges absolutely for all $z \in \mathbb{U}$ and hence represents an analytic function in the unit disk $\mathbb{U}$ (see, for details, [16]). Each of the identities (asserted by Lemma 2.2 below) is well-known.

Lemma 2.2. For real or complex parameters $\alpha_{1}, \alpha_{2}, \beta_{1}\left(\beta_{1} \neq 0,-1,-2, \cdots\right)$, $\mathfrak{R e}\left(\beta_{1}\right)>\mathfrak{R e}\left(\alpha_{2}\right)>0$, we have

$$
\begin{aligned}
& \int_{0}^{1} t^{\alpha_{2}-1}(1-t)^{\beta_{1}-\alpha_{2}-1}(1-t z)^{-\alpha_{1}} d t=\frac{\Gamma\left(\alpha_{1}\right) \Gamma\left(\beta_{1}-\alpha_{1}\right)}{\Gamma\left(\beta_{1}\right)}{ }_{2} F_{1}\left(\alpha_{1}, \alpha_{2} ; \beta_{1} ; z\right) ; \\
& { }_{2} F_{1}\left(\alpha_{1}, \alpha_{2} ; \beta_{1} ; z\right)={ }_{2} F_{1}\left(\alpha_{2}, \alpha_{1} ; \beta_{1} ; z\right) ; \\
& { }_{2} F_{1}\left(\alpha_{1}, \alpha_{2} ; \beta_{1} ; z\right)=(1-z)^{-\alpha}{ }_{2} F_{1}\left(\alpha_{1}, \beta_{1}-\alpha_{2} ; \beta_{1} ; z / z-1\right) ; \\
& \left(\alpha_{1}+1\right){ }_{2} F_{1}\left(1, \alpha_{1} ; \alpha_{1}+1 ; z\right)=\left(\alpha_{1}+1\right)+\alpha_{1} z_{2} F_{1}\left(1, \alpha_{1}+1 ; \alpha_{1}+2 ; z\right) .
\end{aligned}
$$

\section{Main results}

Unless otherwise mentioned, we will suppose in the remainder of this paper that $z \in \mathbb{U}$, the powers are understood as principle values and the parameters $p, m, A, B, \sigma, \alpha_{i}, \beta_{j}, \mu, \lambda_{1}, \lambda_{2}, \ell$, and $d$ are constrained as follows:

$p \in \mathbb{N}, \sigma>0, \mu>-p, \alpha_{i} \in \mathbb{C}(i=1, \ldots, q), \beta_{j} \in \mathbb{C} \backslash\{0,-1,-2, \ldots\}.(j=1, \ldots, s), m, d \in \mathbb{N}_{0}, \lambda_{2} \geqslant \lambda_{1}>0, \ell>0$, such that $\ell p+d>0,-1 \leqslant B<A \leqslant 1$.

Theorem 3.1. Let $\mathrm{f} \in \mathcal{A}_{\mathrm{p}}$, satisfy the following subordination

$$
(1+\beta)\left(\frac{z^{p}}{\mathcal{D}_{\lambda_{1}, \lambda_{2}, \ell, d}^{m, p}\left[\alpha_{i}, \beta_{j}\right] f(z)}\right)^{\sigma}-\beta\left(\frac{z^{p}}{\mathcal{D}_{\lambda_{1}, \lambda_{2}, \ell, d}^{m, p}\left[\alpha_{i}, \beta_{j}\right] f(z)}\right)^{\sigma}\left(\frac{\mathcal{D}_{\lambda_{1}, \lambda_{2}, \ell, d}^{m+1, d}\left[\alpha_{i}, \beta_{j}\right] f(z)}{\mathcal{D}_{\lambda_{1}, \lambda_{2}, \ell, d}^{m, p}\left[\alpha_{i}, \beta_{j}\right] f(z)}\right) \prec \frac{1+A z}{1+B z},
$$

then

$$
\left(\frac{z^{p}}{\mathcal{D}_{\lambda_{1}, \lambda_{2}, \ell, d}^{m, p}\left[\alpha_{i}, \beta_{j}\right] f(z)}\right)^{\sigma} \prec q(z) \prec \frac{1+A z}{1+B z}
$$

where

$$
q(z)=\left\{\begin{array}{ll}
\frac{A}{B}+\left(1-\frac{A}{B}\right)(1+B z)^{-1}{ }_{2} F_{1}\left(1,1 ; \frac{\sigma\left(\ell\left(p+\lambda_{2}(k-p)\right)+d\right)}{\beta \ell \lambda_{1}}+1 ; \frac{B z}{B z+1}\right), & (B \neq 0) \\
1+\frac{\sigma\left(\ell\left(p+\lambda_{2}(k-p)\right)+d\right)}{\sigma\left(\ell\left(p+\lambda_{2}(k-p)\right)+d\right)+\beta \ell \lambda_{1}} A z, & (B=0)
\end{array},\right.
$$


and $\mathrm{q}(z)$ is the best dominant. Furthermore

$$
\mathfrak{R e}\left\{\left(\frac{z^{p}}{\mathcal{D}_{\lambda_{1}, \lambda_{2}, \ell, d}^{m, p}\left[\alpha_{i}, \beta_{j}\right] f(z)}\right)^{\sigma}\right\}>\rho,
$$

where

$$
\rho=\left\{\begin{array}{ll}
\frac{\mathrm{A}}{\mathrm{B}}+\left(1-\frac{\mathrm{A}}{\mathrm{B}}\right)(1-\mathrm{B})^{-1}{ }_{2} \mathrm{~F}_{1}\left(1,1 ; \frac{\sigma\left(\ell\left(p+\lambda_{2}(k-p)\right)+d\right)}{\beta \ell \lambda_{1}}+1 ; \frac{\mathrm{B}}{\mathrm{B}-1}\right), & (\mathrm{B} \neq 0) \\
1-\frac{\sigma\left(\ell\left(p+\lambda_{2}(k-p)\right)+d\right)}{\sigma\left(\ell\left(p+\lambda_{2}(k-p)\right)+d\right)+\beta \ell \lambda_{1}} \mathrm{~A}, & (B=0)
\end{array} .\right.
$$

This result is sharp.

Proof. Set

$$
p(z)=\left(\frac{z^{p}}{\mathcal{D}_{\lambda_{1}, \lambda_{2}, \ell, d}^{m, p}\left[\alpha_{i}, \beta_{j}\right] f(z)}\right)^{\sigma}
$$

then $p(z)$ is of the form (2.1) and is analytic in $\mathbb{U}$. Using (1.7), (3.1) and (3.4) we get

$$
p(z)+\frac{\beta \ell \lambda_{1}}{\left.\sigma\left(\ell\left(p+\lambda_{2}(k-p)\right)+d\right)\right)} z p^{\prime}(z) \prec \frac{1+A z}{1+B z} .
$$

Thus, by Lemma 2.1 for $\gamma=\frac{\sigma\left(\ell\left(p+\lambda_{2}(k-p)\right)+d\right)}{\beta \ell \lambda_{1}}$, we obtain

$$
\begin{aligned}
& \left(\frac{z^{p}}{\mathcal{D}_{\lambda_{1}, \lambda_{2}, \ell, d}^{m, p}\left[\alpha_{i}, \beta_{j}\right] f(z)}\right)^{\sigma} \prec q(z)=\left(\frac{\sigma\left(\ell\left(p+\lambda_{2}(k-p)\right)+d\right)}{\beta \ell \lambda_{1}}\right)
\end{aligned}
$$

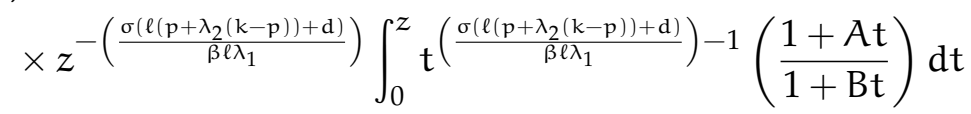

$$
\begin{aligned}
& =\left\{\begin{array}{ll}
\frac{A}{B}+\left(1-\frac{A}{B}\right)(1+B z)^{-1}{ }_{2} F_{1}\left(1,1 ; \frac{\sigma\left(\ell\left(p+\lambda_{2}(k-p)\right)+d\right)}{\beta \ell \lambda_{1}}+1 ; \frac{B z}{B z+1}\right), & (B \neq 0) \\
1+\frac{\sigma\left(\ell\left(p+\lambda_{2}(k-p)\right)+d\right)}{\sigma\left(\ell\left(p+\lambda_{2}(k-p)\right)+d\right)+\beta \ell \lambda_{1}} A z, & (B=0)
\end{array},\right.
\end{aligned}
$$

by change of variables followed by the use of the identities (2.3),(2.4) and (2.5) from Lemma 2.1 with $\alpha_{1}=1, \beta_{1}=\alpha_{2}+1, \alpha_{2}=\frac{\sigma\left(\ell\left(p+\lambda_{2}(k-p)\right)+d\right)}{\beta \ell \lambda_{1}}$. This proves the assertion (3.2). Following the same lines as in Theorem 4 [12], we can prove that $\inf \{\mathfrak{R e}(\mathbf{q}(z))\}=q(-1)$. The proof of Theorem 3.1 is thus completed.

Theorem 3.2. Let $\mathrm{f} \in \mathcal{A}_{\mathrm{p}}$, satisfy the following subordination

$$
(1+\beta)\left(\frac{z^{p}}{\mathcal{D}_{\lambda_{1}, \lambda_{2}, \ell, d}^{m, p}\left[\alpha_{i}, \beta_{j}\right] f(z)}\right)^{\sigma}-\beta\left(\frac{z^{p}}{\mathcal{D}_{\lambda_{1}, \lambda_{2}, \ell, d}^{m, p}\left[\alpha_{i}, \beta_{j}\right] f(z)}\right)^{\sigma}\left(\frac{\mathcal{D}_{\lambda_{1}, \lambda_{2}, \ell, d}^{m, p}\left[\alpha_{i}+1, \beta_{j}\right] f(z)}{\mathcal{D}_{\lambda_{1}, \lambda_{2}, \ell, d}^{m, p}\left[\alpha_{i}, \beta_{j}\right] f(z)}\right) \prec \frac{1+A z}{1+B z},
$$

then

$$
\left(\frac{z^{p}}{\mathcal{D}_{\lambda_{1}, \lambda_{2}, \ell, d}^{m, p}\left[\alpha_{i}, \beta_{j}\right] f(z)}\right)^{\sigma} \prec q(z) \prec \frac{1+A z}{1+B z}
$$

where

$$
q(z)=\left\{\begin{array}{ll}
\frac{A}{B}+\left(1-\frac{A}{B}\right)(1+B z)^{-1}{ }_{2} F_{1}\left(1,1 ; \frac{\sigma \alpha_{i}}{\beta}+1 ; \frac{B z}{B z+1}\right), & (B \neq 0) \\
1+\frac{\sigma \alpha_{i}}{\sigma \alpha_{i}+\beta} A z, & (B=0)
\end{array},\right.
$$

and $\mathrm{q}(z)$ is the best dominant. Furthermore

$$
\mathfrak{R e}\left\{\left(\frac{z^{\mathrm{p}}}{\mathcal{D}_{\lambda_{1}, \lambda_{2}, \ell, \mathrm{d}}^{\mathrm{m}, \mathrm{d}}\left[\alpha_{i}, \beta_{j}\right] f(z)}\right)^{\sigma}\right\}>\mathrm{k},
$$


where

$$
\kappa=\left\{\begin{array}{ll}
\frac{A}{B}+\left(1-\frac{A}{B}\right)(1-B)^{-1}{ }_{2} F_{1}\left(1,1 ; \frac{\sigma \alpha_{i}}{\beta}+1 ; \frac{B}{B-1}\right), & (B \neq 0) \\
1-\frac{\sigma \alpha_{i}}{\sigma \alpha_{i}+\beta} A, & (B=0)
\end{array} .\right.
$$

This result is sharp.

Proof. Set

$$
p(z)=\left(\frac{z^{p}}{\mathcal{D}_{\lambda_{1}, \lambda_{2}, \ell, d}^{m, p}\left[\alpha_{i}, \beta_{j}\right] f(z)}\right)^{\sigma}
$$

then $p(z)$ is of the form (2.1) and is analytic in $\mathbb{U}$. Differentiating both sides of (3.8), and using (1.8), we have

$$
p(z)+\frac{\beta}{\sigma \alpha_{i}} z p^{\prime}(z) \prec \frac{1+A z}{1+B z} .
$$

Now the remaining part of the proof follows by employing the techniques that we used in proof of Theorem 3.1.

Theorem 3.3. Let $\mathrm{f} \in \mathcal{A}_{\mathrm{p}}$, satisfy the following subordination

$$
(1+\beta)\left(\frac{z^{\mathrm{p}}}{\mathcal{D}_{\lambda_{1}, \lambda_{2}, \ell, \mathrm{d}}^{\mathrm{m}, \mathrm{p}}\left[\alpha_{i}, \beta_{\mathrm{j}}\right] \mathrm{I}_{\mu, \mathrm{p}}(z)}\right)^{\sigma}-\beta\left(\frac{z^{\mathrm{p}}}{\mathcal{D}_{\lambda_{1}, \lambda_{2}, \ell, \mathrm{d}}^{\mathrm{m}, \mathrm{p}}\left[\alpha_{i}, \beta_{j}\right] \mathrm{I}_{\mu, \mathrm{p}}(z)}\right)^{\sigma+1}\left(\frac{z^{\mathrm{p}}}{\mathcal{D}_{\lambda_{1}, \lambda_{2}, \ell, \mathrm{d}}^{\mathrm{m}, \mathrm{p}}\left[\alpha_{i}, \beta_{\mathrm{j}}\right] \mathrm{f}(z)}\right)^{-1} \prec \frac{1+A z}{1+\mathrm{Bz}},
$$

then

$$
\left(\frac{z^{p}}{\mathcal{D}_{\lambda_{1}, \lambda_{2}, \ell, d}^{m, p}\left[\alpha_{i}, \beta_{j}\right] I_{\mu, p}(z)}\right)^{\sigma} \prec q(z) \prec \frac{1+A z}{1+B z},
$$

where $\mathrm{I}_{\mu, \mathrm{p}}$ is defined by (1.9) and $\mathrm{q}(z)$ is given by

$$
q(z)=\left\{\begin{array}{ll}
\frac{A}{B}+\left(1-\frac{A}{B}\right)(1+B z)^{-1}{ }_{2} F_{1}\left(1,1 ; \frac{\sigma(\mu+p)}{\beta}+1 ; \frac{B z}{B z+1}\right), & (B \neq 0) \\
1+\frac{\sigma(\mu+p)}{\sigma(\mu+p)+\beta} A z, & (B=0)
\end{array},\right.
$$

and $\mathrm{q}(z)$ is the best dominant. Furthermore

$$
\mathfrak{R e}\left\{\left(\frac{z^{\mathrm{p}}}{\mathcal{D}_{\lambda_{1}, \lambda_{2}, \ell, \mathrm{d}}^{\mathrm{m}, \mathrm{d}}\left[\alpha_{i}, \beta_{j}\right] \mathrm{I}_{\mu} \mathrm{f}(z)}\right)^{\sigma}\right\}>\xi,
$$

where

$$
\xi=\left\{\begin{array}{ll}
\frac{A}{B}+\left(1-\frac{A}{B}\right)(1-B)^{-1}{ }_{2} F_{1}\left(1,1 ; \frac{\sigma(\mu+p)}{\beta}+1 ; \frac{B}{B-1}\right), & (B \neq 0) \\
1-\frac{\sigma(\mu+p)}{\sigma(\mu+p)+\beta} A, & (B=0)
\end{array} .\right.
$$

This result is sharp.

Proof. Set

$$
p(z)=\left(\frac{z^{p}}{\mathcal{D}_{\lambda_{1}, \lambda_{2}, \ell, d}^{m, p}\left[\alpha_{i}, \beta_{j}\right] \mathrm{I}_{\mu, p}(z)}\right)^{\sigma},
$$

then $p(z)$ is of the form (2.1) and is analytic in $\mathbb{U}$. Differentiating both sides of (3.12), and using (1.10), we have

$$
p(z)+\frac{\beta}{\sigma(\mu+p))} z p^{\prime}(z) \prec \frac{1+A z}{1+B z} .
$$


Using Lemma 2.2 for $\gamma=\frac{\sigma(\mu+p)}{\beta}$, we obtain

$$
\begin{aligned}
p(z) \prec q(z) & =\left(\frac{\sigma(\mu+p)}{\beta}\right) z^{-\left(\frac{\sigma(\mu+p)}{\beta}\right)} \int_{0}^{z} t^{\left(\frac{\sigma(\mu+p)}{\beta}\right)-1}\left(\frac{1+A t}{1+B t}\right) d t \\
& = \begin{cases}\frac{A}{B}+\left(1-\frac{A}{B}\right)(1+B z)^{-1}{ }_{2} F_{1}\left(1,1 ; \frac{\sigma(\mu+p)}{\beta}+1 ; \frac{B z}{B z+1}\right), & (B \neq 0) \\
1+\frac{\sigma(\mu+p)}{\sigma(\mu+p)+\beta} A z, & (B=0)\end{cases}
\end{aligned}
$$

The remaining part of the proof in Theorem 3.3 is similar to that of Theorem 3.1 and so we omit it.

Now we prove the partial converse of Theorem 3.3, for $A=1-2 \rho, 0 \leqslant \rho<1$ and $B=-1$.

Theorem 3.4. If $\mathrm{f} \in \mathcal{A}_{\mathrm{p}}$, satisfies

$$
\mathfrak{R e}\left\{\left(\frac{z^{p}}{\mathcal{D}_{\lambda_{1}, \lambda_{2}, \ell, d}^{m, p}\left[\alpha_{i}, \beta_{j}\right] I_{\mu, p}(z)}\right)^{\sigma}\right\}>\rho, \quad 0 \leqslant \rho<1,
$$

then

$$
\begin{aligned}
\mathfrak{R e}\left\{(1+\beta)\left(\frac{z^{\mathrm{p}}}{\mathcal{D}_{\lambda_{1}, \lambda_{2}, \ell, \mathrm{d}}^{\mathrm{m}, \mathrm{p}}\left[\alpha_{i}, \beta_{\mathrm{j}}\right] \mathrm{I}_{\mu, \mathrm{p}}(z)}\right)^{\sigma}-\right. \\
\left.\quad \beta\left(\frac{z^{\mathrm{p}}}{\mathcal{D}_{\lambda_{1}, \lambda_{2}, \ell, \mathrm{d}}^{\mathrm{m}, \mathrm{d}}\left[\alpha_{i}, \beta_{\mathrm{j}}\right] \mathrm{I}_{\mu, \mathrm{p}}(z)}\right)^{\sigma+1}\left(\frac{z^{\mathrm{p}}}{\mathcal{D}_{\lambda_{1}, \lambda_{2}, \ell, \mathrm{d}}^{\mathrm{m}, \mathrm{d}}\left[\alpha_{i}, \beta_{j}\right] \mathrm{f}(z)}\right)^{-1}\right\}>\rho,|z|>R,
\end{aligned}
$$

where

$$
R=\frac{\sqrt{\beta^{2}+[\sigma(\mu+p)]^{2}}-\beta}{\sigma(\mu+p)}
$$

The bound $R$ is the best possible.

Proof. From (3.13), we have

$$
\left(\frac{z^{p}}{\mathcal{D}_{\lambda_{1}, \lambda_{2}, \ell, d}^{m, p}\left[\alpha_{i}, \beta_{j}\right] \mathrm{I}_{\mu, p}(z)}\right)^{\sigma}=\rho+(1-\rho) p(z) .
$$

We see that $p(z)$ of the form (2.1) is analytic in $\mathbb{U}$ and $\mathfrak{R}\{\{p(z)\}>0, z \in \mathbb{U}$. Differentiating both sides of (3.15), and using (1.10), we get

$$
\begin{aligned}
& \mathfrak{R e}\left\{(1+\beta)\left(\frac{z^{\mathrm{p}}}{\mathcal{D}_{\lambda_{1}, \lambda_{2}, \ell, \mathrm{d}}^{\mathrm{m}, \mathrm{p}}\left[\alpha_{i}, \beta_{j}\right] \mathrm{I}_{\mu, \mathrm{p}}(z)}\right)^{\sigma}\right. \\
& \left.-\beta\left(\frac{z^{p}}{\mathcal{D}_{\lambda_{1}, \lambda_{2}, \ell, d}^{m}\left[\alpha_{i}, \beta_{j}\right] I_{\mu, p}(z)}\right)^{\sigma+1}\left(\frac{z^{p}}{\mathcal{D}_{\lambda_{1}, \lambda_{2}, \ell, d}^{m, p}\left[\alpha_{i}, \beta_{j}\right] f(z)}\right)^{-1}-\rho\right\} \\
& =(1-\rho) \mathfrak{R e}\left\{p(z)+\frac{\beta}{\sigma(\mu+p)} z p^{\prime}(z)\right\} \\
& \geqslant(1-\rho)\left[\mathfrak{R e}(p(z))-\frac{\beta}{\sigma(\mu+p)}\left|z p^{\prime}(z)\right|\right] \text {. }
\end{aligned}
$$

Now, by applying the well-known estimate (see [10])

$$
\frac{\left|z p^{\prime}(z)\right|}{\mathfrak{R} e(p(z))} \leqslant \frac{2 r}{1-r^{2}}, \quad(|z|=r<1)
$$


in (3.16), we deduce that

$$
\begin{aligned}
\mathfrak{R e}\{(1+\beta) & \left(\frac{z^{\mathrm{p}}}{\mathcal{D}_{\lambda_{1}, \lambda_{2}, \ell, \mathrm{d}}^{\mathrm{m}, \mathrm{p}}\left[\alpha_{i}, \beta_{\mathrm{j}}\right] \mathrm{I}_{\mu, \mathrm{p}}(z)}\right)^{\sigma} \\
& \left.\quad-\beta\left(\frac{z^{\mathrm{p}}}{\mathcal{D}_{\lambda_{1}, \lambda_{2}, \ell, \mathrm{d}}^{\mathrm{m}, \mathrm{p}}\left[\alpha_{i}, \beta_{\mathrm{j}}\right] \mathrm{I}_{\mu, \mathrm{p}}(z)}\right)^{\sigma+1}\left(\frac{z^{\mathrm{p}}}{\mathcal{D}_{\lambda_{1}, \lambda_{2}, \ell, \mathrm{d}}^{\mathrm{m}, \mathrm{d}}\left[\alpha_{i}, \beta_{\mathrm{j}}\right] f(z)}\right)^{-1}-\rho\right\} \\
\geqslant & (1-\rho) \mathfrak{R e}(\mathrm{p}(z))\left(1-\frac{\beta}{2 \mathrm{r} \beta \sigma(\mu+p)\left(1-\mathrm{r}^{2}\right)}\right),
\end{aligned}
$$

which is positive if $r<R$, where $R$ is given by (3.14).

In order to show that the bound $R$ is the best possible, we consider the function $f \in \mathcal{A}_{p}$ defined by

$$
\left(\frac{z^{p}}{\mathcal{D}_{\lambda_{1}, \lambda_{2}, \ell, d}^{m, p}\left[\alpha_{i}, \beta_{j}\right] I_{\mu, p}(z)}\right)^{\sigma}=\rho+(1-\rho) \frac{1+z}{1-z} .
$$

Noting that

$$
\begin{aligned}
& \mathfrak{R e}\{(1+\beta)\left(\frac{z^{\mathrm{p}}}{\mathcal{D}_{\lambda_{1}, \lambda_{2}, \ell, \mathrm{d}}^{\mathrm{m}, \mathrm{p}}\left[\alpha_{j}, \beta_{\mathrm{j}}\right] \mathrm{I}_{\mu, \mathrm{p}}(z)}\right)^{\sigma} \\
&\left.\quad-\beta\left(\frac{z^{\mathrm{p}}}{\mathcal{D}_{\lambda_{1}, \lambda_{2}, \ell, \mathrm{d}}^{\mathrm{m}, \mathrm{d}}\left[\alpha_{i}, \beta_{\mathrm{j}}\right] \mathrm{I}_{\mu, \mathrm{p}}(z)}\right)^{\sigma+1}\left(\frac{z^{\mathrm{p}}}{\mathcal{D}_{\lambda_{1}, \lambda_{2}, \ell, \mathrm{d}}^{\mathrm{m}, \mathrm{d}}\left[\alpha_{i}, \beta_{\mathrm{j}}\right] \mathrm{f}(z)}\right)^{-1}-\rho\right\} \\
&=(1-\rho) \mathfrak{R e}\left(\frac{1+z}{1-z}+\frac{\beta z}{\sigma(\mu+\mathrm{p})} \frac{2}{(1-z)^{2}}\right)=0
\end{aligned}
$$

for $z=R$, we complete the proof of Theorem 3.4.

\section{Acknowledgment}

The second author is supported by UKM grant: GUP-2017-064.

\section{References}

[1] F. M. Al-Oboudi, On univalent functions defined by derivative operator, Inter. J. Math. Math. Sci., 2004 (2004), 14291436. 1.2

[2] A. Căta̧s, On certain classes of p-valent functions defined by new multiplier transformations, In Proceedings of the International Symposium on Geometric Function Theory and Applications: GFTA, (2007), 241-250. 1.2

[3] N. E. Cho, H. M. Srivastava, Argument estimates of certain analytic functions defined by a class of multiplier transformations, Math. Comput. Modelling, 37 (2003), 39-49. 1.2

[4] J. Dziok, H. M. Srivastava, Classes of analytic functions associated with the generalized hypergeometric function, Appl. Math. Comput., 103 (1999), 1-13. 1, 1.2

[5] E. A. Eljamal, M. Darus, Subordination results defined by a new differential operator, Acta Univ. Apulensis Math. Inform., 27 (2011), 121-126. 1.2

[6] E. El-Yaguby, M. Darus, Subclasses of analytic functions defined by new generalized derivative operator, J. Qual. Meas. Anal., 9 (2013), 47-56. 1.2

[7] E. El-Yagubi, M. Darus, M. Aydogan, On a subclass of p-valent starlike functions associated with a generalized hypergeometric differential operator, New Develop. Pure Appl. Math., 159 (2015), 159-162. 1.2

[8] D. J. Hallenbeck, S. Ruscheweyh, Subordination by convex functions, Proc. Amer. Math. Soc., 52 (1975), 191-195. 2.1

[9] S. S. Kumar, H. C. Taneja, V. Ravichandran, Classes multivalent functions defined by DziokSrivastava linear operator and multiplier transformations, Kyungpook Math. J., 46 (2006), 97-109. 1.2

[10] T. H. MacGregor, The radius of univalence of certain analytic functions, Proc. Amer. Math. Soc., 14 (1963), 514-520. 3

[11] A. Oshah, M. Darus, Differential sandwich theorems with a new generalized derivative operator, Adv. Math., Sci. J., 3 (2014), 117-124. 1.2 
[12] J. Patel, N. E. Cho, H. M. Srivastava, Certain subclasses of multivalent functions associated with a family of linear operators, Math. Comput. Model., 43 (2006), 320-338. 3

[13] G. S. Sălăgean, Subclasses of univalent functions, Complex analysis-fifth Romanian-Finnish seminar, Part 1 (Bucharest, 1981), 362-372, Lecture Notes in Math., 1013, Springer, Berlin, (1983). 1.2

[14] S. R. Swamy, Inclusion properties of certain subclasses of analytic functions, Int. Math. Forum, 7 (2012), 1751-1760. 1.2

[15] S. R. Swamy, Inclusion properties of certain subclasses of analytic functions defined by a multiplier transformation, Int. J. Math. Anal. (Ruse), 6 (2012), 1553-1564. 1.2

[16] E. T. Whittaker, G. N. Watson, A Course on Modern Analysis: An Introduction to the General Theory of Infinite Processes and of Analytic Functions; With an Account of the Principal Transcendental Functions, 4th edition, Cambridge University Press, Cambridge, (1927). 2 